\title{
Conhecimento sobre fatores de risco para hipertensão arterial sistêmica por discentes do curso de enfermagem
}

\section{Knowledge of risk factors for systemic arterial hypertension by nursing courses}

\author{
Ayla de Araujo Beserra \\ Graduando em bacharelado de Enfermagem pela a Escola Superior de Saúde Arcoverde- ESSA \\ e-mail: aylla-araujo28@hotmail.com \\ Luiza Vanessa de Lima Silva \\ Graduando em bacharelado de Enfermagem pela a Escola Superior de Saúde Arcoverde- ESSA \\ Emanuel Silva de Miranda \\ Graduando em bacharelado de Enfermagem pela a Escola Superior de Saúde Arcoverde- ESSA \\ E-mail:emanueltrust@hotmail.com \\ Maria Renata de Almeida \\ Graduando em bacharelado de Enfermagem pela a Escola Superior de Saúde Arcoverde- ESSA \\ Silvana Cavalcanti dos Santos \\ Docentee Orientadora pela a ESSA e Msc. Em Saúde Coletiva pela a Fundação Osvaldo Cruz-FIOCRUZ
}

\begin{abstract}
Resumo: Dentre as doenças crônicas não transmissíveis, a hipertensão arterial sistêmica (HAS) é o maior problema de saúde social dos países desenvolvidos e em muitos dos emergentes. Nesse sentindo, objetivou-se identificar os fatores de risco dos discentes de enfermagem para o desenvolvimento da HAS em um centro de ensino superior de saúde na cidade de Arcoverde, Pernambuco. Trata-se de um estudo de caráter descritivo e exploratório com abordagem quantitativa. A amostra foi composta por 33 discentes, que cursavam o primeiro e o sexto período do curso superior de enfermagem. As variáveis estudadas foram: identificações, socioeconômicos e comportamentais. Em relação ao gênero, dos alunos consultados, apresentaram-se em maior porcentagem os do gênero feminino, informando que fazem uso de bebida alcoólica e apresentam hábitos saudáveis de alimentação. Evidenciou-se à inatividade física, sendo o fator mais citado dos riscos modificáveis, e os antecedentes familiares e a raça, fatores estes intrínsecos não modificáveis. Quanto ao nível de conhecimento sobre a HAS, nos dois períodos, é caracterizado como insuficiente sobre a doença hipertensiva. Torna-se necessário aprofundar alguns conhecimentos sobre HAS dos estudantes e a implementação de orientações sobre a prática de atividade física como um fator preventivo para HAS.
\end{abstract}

Palavras-chave: Enfermagem; Hipertensão; Fatores de Risco, Universitários.

Abstract: Among non-communicable chronic diseases (NCCD), Systemic arterial hypertension (SAH) Is the largest social health problem in developed and many emerging countries.In this sense, the objective was to identify the risk factors of nursing students for developmentOf SAH In a center of higher education of health in the city of Arcoverde, Pernambuco.This is a descriptive and exploratory study with a quantitative approach. The sample consisted of 33 students from the first and sixth periods of the nursing course. The studied variables were: identification, socioeconomic and behavioral. Regarding the gender, the students consulted presented a higher percentage of the female gender, a significant number of students reported using alcohol, and presented healthy eating habits. Physical inactivity was the most mentioned factor of the modifiable risks, the family antecedents and the breed, these intrinsic factors not modifiable. The level of knowledge about the SAH of nursing students in both periods is characterized as insufficient on the hypertensive disease.It is necessary to deepen some knowledge about SAH to the students, and the implementation of guidelines on the practice of physical activity as a preventive factor for SAH.

Keywords: Nursing; Hypertension; Risk Factors; University. 


\section{INTRODUÇÃO}

As Doenças Crônicas Não Transmissíveis (DCNT) são consideradas um grande desafio contemporâneo, no contexto das transições epidemiológica, demográfica e nutricional, bem como na globalização dos padrões de consumo, de alimentação e hábitos de vida, que afetam não só o Brasil, mas a maioria dos países do mundo. A Organização Mundial da Saúde (OMS) estima que dos 38 milhões de óbitos ocorridos em 2012 por DCNT, 16 milhões, ou seja, $42 \%$ eram prematuras e evitáveis. Os países de baixa e média renda respondem por cerca de $80 \%$ das mortes no mundo, e acometem em igual número homens e mulheres (OMS, 2011; OMS, 2015).

As DCNT podem causar diversos danos aos pacientes, dentre estes: a diminuição da sua qualidade de vida, produtividade e funcionalidade. Além de apresentar um "fardo" pesado relacionado à morbimortalidade e ao aumento dos custos em saúde, realidade esta preocupante (GOULART, 2011).

Dentre as DCNT, a Hipertensão Arterial Sistêmica (HAS) é o maior problema de saúde social dos países desenvolvidos e em muitos dos países emergentes. No Brasil apresenta alta taxa de prevalência. E, na maioria das vezes, é diagnosticada de forma tardia. Por mais que existam medidas preventivas e de controle, sejam elas ou não farmacológicos, ela continua a ser um dos maiores desafios em saúde (SANTOS, 2011).

Conforme dados da Organização Mundial de Saúde (OMS), mais de 9,4 milhões de pessoas têm morrido por causa de hipertensão. No Brasil, o Ministério da Saúde relata que, aproximadamente, 300 mil óbitos ocorrem por ano, 820 mortes por dia, 30 por hora ou uma a cada 2 minutos (BRASIL, 2016; SBH, 2015). Em torno de uma a cada quatro pessoas adultas tem adquirido HAS. Avaliando-se que a HAS atinge, aproximadamente, $30 \%$ da população brasileira adulta, chegando a mais de $50 \%$ após os 60 anos. E está presente em 5\% das crianças e adolescentes. É responsável por $40 \%$ dos infartos, $80 \%$ dos derrames e $25 \%$ dos casos de insuficiência renal terminal (SBH; SBC, 2015).

A HAS é uma síndrome multifatorial, multicausal e multisistêmica. Seu surgimento está ligado a um estilo de vida inapropriado, levando em consideração os fatores de risco não modificáveis, como: sexo, idade, raça/cor e história familiar, e os fatores de risco modificáveis, como: sedentarismo, estresse, tabagismo, alcoolismo, alimentação e obesidade (SANTOS, 2011).

A HAS é uma condição clínica relacionada com diversos fatores, caracterizada por níveis pressóricos iguais ou maiores que $140 \mathrm{mmHg}$ na sistólica e $90 \mathrm{mmHg}$ na diastólica. Uma vez presente e não tratada pode levar a alterações funcionais e/ou estruturais em órgãos alvo (coração, encéfalo, rins e vasos sanguíneos) e alterações metabólicas, aumentando o risco para problemas cardiovasculares fatais e não fatais (SBH; SBC; SBN, 2010). A Hipertensão é um fator de risco para diversas doenças como: aterosclerose, trombose (que levam a isquemia de diversos órgãos), Alzheimer e demência vascular. Portanto, torna-se precursora de muitas doenças crônicas não transmissíveis, levando a uma redução de expectativa e qualidade de vida (DUNCAN et al., 2004).
No Brasil e no mundo vem se empreendendo esforços para melhorar a integridade e a exatidão das estatísticas vitais, juntamente com o lançamento de novos métodos e abordagens para monitoramento das DCNT e dos fatores de risco comportamentais (MINAYO; GUALHANO, 2015; OMS, 2011). Em setembro de 2011, a Organização das Nações Unidas (ONU) realizou uma reunião com o objetivo de estabelecer compromissos dos chefes de Estados para o enfrentamento das DCNT, por meio de ações de prevenção dos seus principais fatores de risco e garantia de atenção adequada à saúde dos portadores dessas patologias (ONU, 2011).

Nos últimos anos, a população jovem vem apresentando alta taxa de prevalência em HAS, essa realidade é preocupante, pois os níveis pressóricos estão aumentados e tendem a permanecer na fase adulta, levando o aparecimento de complicações precoces, mostrando a necessidade de práticas preventivas e educativas (COSTA, 2012). É importante que os níveis pressóricos sejam aferidos nessa fase, pois é nela que, muitas vezes, ocorre o desenvolvimento inicial da hipertensão (BARRETO NETO, 2010).

Diante do exposto, objetivou-se identificar os fatores de risco dos discentes de enfermagem para o desenvolvimento da hipertensão arterial sistêmica em um centro de ensino superior de saúde na cidade de Arcoverde, Pernambuco.

\section{MATERIAL E MÉTODOS}

Trata-se de um estudo de caráter descritivo e exploratório com abordagem quantitativa. Pesquisa realizada com os discentes de enfermagem de um centro particular de ensino superior de saúde na cidade de Arcoverde, no Estado de Pernambuco. A pesquisa foi realizada em setembro de 2015 com 33 discentes do curso de Enfermagem, destes 12 cursavam o primeiro período e 21 cursavam o sexto período. Foram adotados os seguintes critérios de inclusão: (a) está matriculado no curso de enfermagem no primeiro ou sexto períodos; b) ter assinado o Termo de Consentimento Livre Esclarecido.

Os dados foram coletados através de um questionário, elaborado pelos autores/pesquisadores, contendo questões objetivas e subjetivas. No qual foi realizado um validação do instrumento de pesquisa e suas possíveis correções. Foram adotas as variáveis: identificações, socioeconômicos e comportamentais.

Para a realização da aferição da Pressão Sanguínea (PA) dos discentes e dos seus níveis pressóricos, foram adotados como base os valores de referência para os limites de uma PA normal e de seus valores pressóricos da Hipertensão Arterial Sistêmica (HAS) (SBH; SBC; SBN, 2010), no laboratório do centro com duas aferições da PA, uma antes da entrevista e uma depois da entrevista.

Os resultados são apresentados por meio de tabelas e gráficos elaborados nos programas Microsoft Word e Microsoft Excel, em termos absolutos e percentuais.

O estudo foi submetido ao Comitê de Ética em Pesquisa segundo o Conselho Nacional de Saúde através da Resolução n 466/12 (BRASIL, 2012) e da Autarquia Educacional de Belo Jardim (AEB), tendo sido realizado após a sua aprovação, sob o Parecer $n^{\circ} 1.209 .224,01$ de setembro de 2015 . 


\section{RESULTADOS E DISCUSSÃO}

Na Tabela 1, observa-se a caracterização dos estudantes quanto ao gênero, raça e nível de conhecimento sobre a HAS. Em relação ao gênero, nos dois períodos, apresentaram uma maior porcentagem de universitários do sexo feminino, respectivamente $75 \%$ no primeiro período e $95,20 \%$ no sexto período. Dado observado também em outros estudos com discentes do curso de enfermagem, à maioria era do sexo feminino (BERNARDES et al., 2015; FREITAS et al., 2012). Os homens e as mulheres apresentam quase a mesma taxa de prevalência de hipertensão arterial sistêmica, sendo superior nos homens até os 50 anos e superior nas mulheres a partir dos 50 anos (WESCHENFELDER; GUE, 2012).

Tabela 1. Distribuição da Amostra conforme a raça, gênero e nível de conhecimento da HAS em centro de ensino superior na cidade de Arcoverde, Pernambuco $(n=33)$.

\begin{tabular}{|c|c|c|c|c|}
\hline \multirow{2}{*}{$\begin{array}{l}\text { Variáveis } \\
\text { Sexo }\end{array}$} & \multicolumn{2}{|c|}{$\begin{array}{c}\text { Primeiro período } \\
n^{\mathbf{0}} \mathbf{1 2}\end{array}$} & \multicolumn{2}{|c|}{$\begin{array}{c}\text { Sexto período } \\
n^{\mathbf{0}} 21 \\
\end{array}$} \\
\hline & $\mathbf{N}^{\mathbf{0}}$ & $\%$ & $\mathbf{N}^{\mathbf{o}}$ & $\%$ \\
\hline Feminino & 03 & 25,00 & 01 & 4,80 \\
\hline Masculino & 09 & 75,00 & 20 & 95,20 \\
\hline \multicolumn{5}{|l|}{ Raça } \\
\hline Branca & 04 & 33,34 & 08 & 38,10 \\
\hline Parda & 08 & 66,66 & 13 & 61,90 \\
\hline \multicolumn{5}{|l|}{ Conceito HAS } \\
\hline $\mathrm{DCNT} / \mathrm{DC}$ & 11 & 91,70 & 19 & 90,50 \\
\hline D. Degenerativa & 01 & 08,30 & 02 & 09,50 \\
\hline \multicolumn{5}{|l|}{ Valor de referência $\mathbf{P A}$} \\
\hline $120 / 80 \mathrm{mmHg}$ & 10 & 83,40 & 07 & 33,34 \\
\hline $140 / 90 \mathrm{mmHg}$ & 02 & 16,60 & 14 & 66,66 \\
\hline \multicolumn{5}{|l|}{ Órgãos Alvos / Coração/Rins e Cérebro } \\
\hline Acertos & 09 & 75,00 & 21 & 100,00 \\
\hline Erros & 03 & 25,00 & - & - \\
\hline \multicolumn{5}{|l|}{ Complicações/HAS } \\
\hline IAM/AVE & 04 & 33,34 & 14 & 66,66 \\
\hline Outros (Tonturas/Vertigens/Dor de Cabeça) & 08 & 66,66 & 07 & 33,34 \\
\hline \multicolumn{5}{|c|}{ Prevalência da HAS (Considerando os Acertos) } \\
\hline Em Negros & 03 & 25,00 & 13 & 61,90 \\
\hline Em Diabéticos & 10 & 83,40 & 17 & 80,96 \\
\hline Medicação Para HAS que Causa AVE & 06 & 50,00 & 13 & 61,90 \\
\hline \multicolumn{5}{|l|}{ Medidas Prevenção Para HAS } \\
\hline Atividade Física/Álimentação Saudável & 10 & 83,40 & 16 & 76,20 \\
\hline $\begin{array}{l}\text { Outras respostas } \\
\text { (Beber água, Toar Medicação) }\end{array}$ & 02 & 16,60 & 05 & 3,80 \\
\hline
\end{tabular}

Quanto à raça, 66,66\% do primeiro período e $61,90 \%$ do sexto período se consideram de cor parda. A hipertensão é duas vezes mais prevalente nas pessoas de cor não branca (LESSA, 2001). Segundo Baldinho (2014), em estudo realizado sobre a influência da raça na HAS, observou-se que, entre toda população da amostra com a doença, $61,8 \%$ eram pretos e $48,8 \%$ pardos.

Em relação aos conhecimentos dos estudantes sobre a categoria da hipertensão arterial como uma patologia, verificou-se que $97,70 \%$ do primeiro período e $90,50 \%$ do sexto período, a caracterizaram como uma doença crônica, assinalaram que ela é uma doença crônica. Santos (2011) afirma que dentre as doenças crônicas não transmissíveis está incluída a hipertensão arterial sistêmica.

Para o valor de referência para hipertensão arterial, $83,40 \%$ dos estudantes do primeiro período erraram ao relatarem que o valor para hipertensão arterial seria de 120x80 mmHg. Este número tão expressivo pode está relacionado à ausência de disciplinas sobre esta temática no módulo. Os estudantes do sexto período apresentaram melhor desempenho, sendo que $66,66 \%$ dos alunos acertaram o valor de referência. Essa taxa de acerto encontrada é similar a outro estudo com alunos de enfermagem, que tinham $54,8 \%$ de conhecimento dos níveis de HAS (OLIVEIRA; GUSMÃO, 2013). A hipertensão arterial sistêmica é uma condição clínica que se caracteriza pelo sustentado e alto nível da pressão arterial de 140 x 90mmHg (SBH; SBC; SBN, 2010). Nota-se que uma parcela significativa dos alunos do sexto período, não acertaram, tornando-se um fato preocupante uma vez que estão próximos de concluir o curso superior de enfermagem e irão trabalhar nos serviços de saúde e em inúmeras campanhas apresentarão esses valores para sociedade (OLIVEIRA; GUSMÃO, 2013).

Constatou-se que a maior parte dos estudantes acertaram quais são os órgãos alvos acometidos pela HAS. O primeiro período com $75 \%$, e o sexto com $100 \%$. A hipertensão arterial está associada ao surgimento de mudanças funcionais e/ou estruturais nos órgãos alvos: coração, encéfalo e rins (SBH; SBC; SBN, 2010).

Quando questionados sobre as principais complicações da hipertensão arterial, 66,66\% dos alunos, do primeiro período, não conseguem diferenciá-las dos sinais e sintomas. Enquanto que, no sexto período, $66,66 \%$ dos estudantes identificaram o acidente vascular encefálico e o infarto agudo do miocárdio como principais 
complicações. É sabido que a HAS é o principal fator de risco para o acidente vascular encefalite (AVE) e o infarto agudo do miocárdio (IAM) (BRASIL, 2006). Na HAS o coração sofre uma sobrecarga, pouco a pouco, tornando-se insuficiente, podendo surgir sinais de sofrimento cerebral. A HAS é um fator de risco para a ocorrência de AVC, devido a um suprimento sanguíneo inadequado (LIMA et al, 2006).

Quanto a assertiva, que afirma que prevalência de HAS é duas vezes maior em diabéticos, observou-se que $83,40 \%$ do primeiro e $80,96 \%$ do sexto período, concordaram com a afirmativa. Segundo a SBC (2015), a HAS em pacientes diabéticos tem uma prevalência duas vezes maior que a população geral. A diabetes está associada a HAS, sendo o hiperinsulinismo e a insulinoresistência causadores do aumento da reabsorção de sódio, aumento da contractilidade, da hipertrofia do tecido muscular liso vascular, do aumento da resposta simpática e disfunção endotelial.

Os alunos foram questionados a respeito de alguns medicamentos para o tratamento da HAS que causariam AVE. Destes, metade dos alunos do primeiro período $50 \%$ e $61,90 \%$ do sexto período afirmaram, que esses medicamentos causam maior risco para AVE. A hipertensão é o maior fator de risco para AVE. E estão indicados para o tratamento os inibidores da ECA, antagonistas dos canais de cálcio e os diuréticos (SBC, 2015).
As medidas de prevenção mais citadas foram uma alimentação saudável e atividades físicas, com porcentagem de $83,40 \%$ no primeiro e $76,20 \%$ no sexto. São inúmeras as orientações para prevenção da HAS dentre elas: acompanhamento contínuo da PA; praticar exercícios físicos; evitar alimentos gordurosos; evitar bebidas alcoólicas; não fumar; evitar estresse; e não interromper o tratamento com remédios sem a orientação médica (SBEM, 2015). Deve-se ressaltar que apesar dos discentes mencionar tais práticas, uma parcela significativa não referencia. Isso vem demonstrar a necessidade da programação e implementação de ações educativas que visem informar ao indivíduo sobre a necessidade da prática de prevenção.

Em relação ao gênero, nos dois períodos apresentaram, uma maior porcentagem de universitários do sexo feminino, respectivamente $75 \%$ no primeiro e $95,20 \%$ no sexto.

Na Figura 1, observa-se os dados referentes ao estilo de vida, sendo que quanto ao Tabagismo, apenas $3 \%$ relatam fazer uso regularmente do cigarro, este dado é bem positivo, uma vez que o fumo é um grande gerenciador de doenças cardiovasculares. Resultados semelhantes foram constatados com 300 discentes de uma universidade pública do sul do país, sendo encontrada uma taxa de prevalência baixa de apenas $4,3 \%$ de tabagismo entre os universitários, independente do sexo (RADOVANOVIC et al., 2012).

Figura 1. Estilo de vida de alunos do curso de enfermagem de um centro de ensino superior na cidade de Arcoverde, Pernambuco.

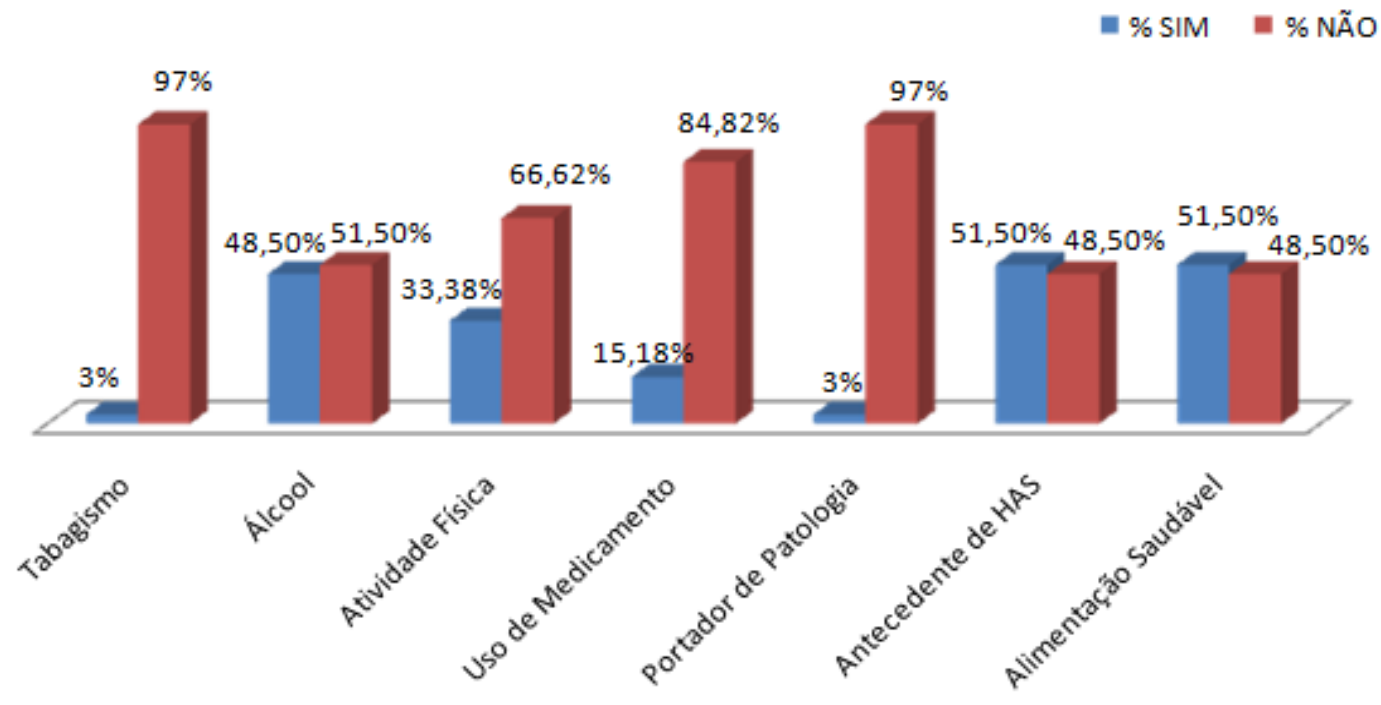

O resultado do presente estudo aponta que 48,50\% dos estudantes fazem uso de bebidas alcoólicas. É sabido que o excesso de álcool no organismo está ligado à elevação do risco de alguns tipos de câncer, distúrbios no fígado e algumas doenças cardiovasculares o que torna este dado preocupante (RADOVANOVIC et al., 2012). Carvalho et al. (2011), estudando os hábitos alcoólicos de acadêmicos, verificou que $8 \%$ apresentavam um elevado consumo, problemas físico-psíquicos em associação ao álcool, e $28 \%$ em risco de vida devido a ingestão de álcool.

Para atividade física, $66,62 \%$ consideraram-se sedentários, o que resulta em um dado preocupante, uma vez que a atividade física é um fator protetor contra aHAS. Assim demonstrando a necessidade da implementação de ações educativas que visem informar ao indivíduo sobre a necessidade da prática do exercício físico. Também é apontado por outras pesquisas feitas com acadêmicos com percentuais de inatividades físicas entre $38 \%$ a 77,20\% (CASTRO et al., 2015; SOAR, 2012). A prática regular de atividade física é um fator protetor para hipertensão. (JACKSON et al., 2014; BERNARDO et al., 2013; ZHANG et al., 2010).

Quanto ao uso de medicação $15,18 \%$ afirmaram que fazem uso, porém essa prática pode, muitas vezes, está camuflando os sinais e sintomas da HAS em relação à 
automedicação. Segundo Lopes et al., (2014b) o indicie de auto medicação entre acadêmicos de odontologia foi de $(94,64 \%)$, radiologia $(68,06 \%)$, direito $(89,80 \%)$, e enfermagem $(94,12 \%)$, não conferida com presente estudo. Entre os argumentos que identificam a automedicação, a idade pode ajudar nesta prática, quando esta pesquisa é constituída por jovens com mediana de idade entre 17 e 18 anos, assimilando aos resultados de Fontanella et al., (2013).

Referente a portar alguma patologia, 3\% dos alunos apresentam. Porém, para Lopes (2014), em casos de HAS secundária, pode existir uma herança genética. Nesses casos podemos destacar a síndrome de Liddle, síndrome do excesso aparente de mineralocorticoide, hipertensão exacerbada pela gravidez, síndrome de Gordon, aldosteronismo suprimível por glicocorticoide, hiperaldosteronismo familiar tipo 2 e hiperplasia adrenal congênita.

Quanto a antecedentes familiares $51,50 \%$ dos estudantes possuem na família algum caso. Esse fator deve ser levado em consideração nos estudos sobre prevalência da HAS. Pois é uma doença na qual o componente genético-hereditário tem grande importância. Neste contexto, o primeiro fator de risco que deve ser evidenciado é a historiografia familiar, associado à HAS, sendo que esse é um fator que pode atuar na progenitura da doença, principalmente em atuação das condições ambientais e culturais do ciclo familiar (GUPTA, KAPOOR, 2013). Dessa forma, verifica-se a necessidade de uma avaliação criteriosa não só quanto aos estilos de vida, também quanto a uma conexão genética em uma família portadora de DCNT.

A alimentação saudável dos estudantes de enfermagem foi caracterizada por $51,50 \%$, pois eles apresentaram uma alimentação regular e saudável, o que gera um grande coeficiente de proteção contra a HAS. Segundo a Sociedade Brasileira de Hipertensão, (2010), uma das medidas preventivas para tratar a HAS muito importante, são as relacionadas mudanças nos padrões de vida. Desta forma, teria uma maior chance de detectar os fatores de risco ligado à doença, o que propicia bons resultados no progresso saudável do indivíduo.

$\mathrm{Na}$ Tabela 2, verifica-se a classificação dos níveis pressóricos. Em associação aos níveis pressóricos, apenas 3\% dos estudantes, demonstraram índices limítrofes - 130 x $80 \mathrm{mmHg}$, sendo esta margem do gênero masculino.

Tabela 2. Distribuição dos estudantes de enfermagem conforme a classificação da Sociedade Brasileira de Cardiologia, (2012) da pressão arterial (PA) em um centro de ensino superior na cidade de Arcoverde, Pernambuco

\begin{tabular}{lcc}
\hline Classificação da PA & $\mathrm{N}=33$ & $\%$ \\
\hline Ótima & 32 & 97 \\
Limítrofe & 1 & 3 \\
Hipertensão Estágio 1 & - & - \\
Hipertensão Estágio 2 & - & - \\
Hipertensão Estágio 3 & - & - \\
\hline Total & 33 & 100 \\
\hline
\end{tabular}

Em outras pesquisas foi observado que os homens apresentam uma variação da pressão arterial (PA) não controlada em análogo às mulheres. Dessa forma, pode-se considerar que a condição feminina teve ação protetora (MARTINS et al., 2010; SILVA et al., 2016).

Vale evidenciar a necessidade de intensificar a abordagem preventiva das DCNT na instituição de ensino superior. Criar espaços que estimule a promoção da saúde dos discentes, dentre estas: o desenvolvimento de atividade física na IES. Mesmo não encontrando nenhuma alteração pressórica nos discentes, parcela significativa dos mesmos apresentam hábitos de vida inapropriados e conhecimento insatisfatório, predispondo ao desenvolvimento de HAS. Situação preocupante visto que o grupo será disseminador de um estilo de vida saudável, como futuros profissionais da área de saúde.

É importante que exista a união de instituições em âmbito político, educacional e de saúde para juntar esforços voltados para prevenção, promoção da saúde, programas de incentivos para políticas regulares de exercício físico e de alimentação saudável, programas educativos que motivem e fortaleçam a importância de mudanças comportamentais para melhor qualidade de vida.

Torna-se necessário aprofundar alguns conhecimentos sobre HAS aos estudantes e a implementação de orientações de educação em saúde acerca dos fatores de risco para hipertensão arterial, com objetivo de modificar os fatores preveníveis. Pois, Tratase de uma doença crônica sem cura e com grande impacto para o sistema de saúde.

\section{CONCLUSÕES}

O nível de conhecimento sobre a HAS dos estudantes de Enfermagem, nos dois períodos, é caracterizado como insuficiente sobre a doença hipertensiva, principalmente, os discentes do sexto período.

\section{REFERÊNCIAS}

BALDINO, A. Hipertensão arterial, complicações cardiovasculares e causas de óbitos na população negra: Estudo de coorte 1983-2014. (Dissertação) Mestrado em Enfermagem. Universidade de Guarulhos. Guarulhos. 2014. 83p.

BARRETO, A. C.; ARAÚJO, E. C.; SILVA, K. V. P.; PONTES, L. M. Prevalência de hipertensão e Fatores associados em adolescentes Escolares no Sertão de Pernambuco. Adolesc. Saude, Rio de Janeiro, v. 7, n 4, p. 21-29. 2010.

BERNARDO, A. F. B.; ROSSI, R. C.; SOUZA, N. M.; PASTRE, C. M.; VANDERLEI, L. C. M. Associação entre atividade física e fatores de risco cardiovasculares em indivíduos de um programa de reabilitação cardíaca. Rev. Brasileira de Medicina do Esporte, São Paulo, v. 19, n. 4, 2013.

BERNARDES, L. E.; VIEIRA, E. E. S.; LIMA, L. H. O.; CARVAlHO, G. C. N.; SILVA, A. R. V.; Os fatores de risco para doenças crônicas não transmissíveis em universitários. Cienc Cuid Saude; v 14 n 2. p:1122-1128. 2015. 
BRASIL. Conselho Nacional de Saúde Resolução No 466, de 12 de dezembro de 2012. Disponível em: http://conselho.saude.gov.br/resolucoes/2012/Reso466.pdf Acesso em: 15.05. 2015.

BRASIL. Ministério da Saúde. Biblioteca Virtual em Saúde. Dia Nacional de Combate a hipertensão arterial. Disponível em: <http://bvsms.saude.gov.br/ultimasnoticias/2268-26-4-dia-nacional-de-prevencao-e-combatea-hipertensao-arterial.> Acesso em agosto de 2016.

BRASIL. Ministério da Saúde. Plano de ações estratégicas para o enfrentamento das doenças crônicas não transmissíveis (DCNT) no Brasil, 2011-2022. Brasília: Ministério da Saúde; 2011. Disponível em: http://bvsms.saude.gov.br/bvs/publicacoes/plano_acoes_e nfrent_dent_2011.pdf. Acesso em: 20 julho. 2015.

BRASIL. Ministério da Saúde Caderno de Atenção Básica: 15. Hipertensão Arterial Sistêmica. Brasília 2006. Disponível

em:

<http://189.28.128.100/dab/docs/publicacoes/cadernos_ab /abcad15.pdf.> Acesso em agosto de 2016.

CARVALHO, A. L.; GOMES, R .I. B.; SOUSA, V. E. X.; SARDINHA, A. H. L.; FILHO, M. C. R. Hábitos alcoólicos entre universitários de uma instituição pública. Rev . Cienc Cuid Saúde. Maringá, v.10, n.3, 2011.

CASTRO,Y. P. P.; VIANA, L. V. M.; SILVA, M. G. P.; AMORIM , L. M. M.; CARVALHO, M. L.; ROCHA, F. C. G. Fatores de risco para hipertensão arterial em acadêmicos de enfermagem de uma universidade. Revista Interdisciplinar. v.8, n.3, 2012.

COSTA, J. V.; SILVA, A. R. V.; MOURA, I. H.; CARVALHO, R. B. N.; BERNANDES, L. E. Analise de Fatores de Risco para Hipertensão Arterial em adolescentes escolares. Rev. Latino-Am. Enfermagem, São Paulo, v. 20, n. 2, 2012.

DUNCAN, B.; SCHMIDT, M. I.; GIUGLIANI, E. R. J. Medicina Ambulatorial: Condutas de atenção Primária baseada em Evidências. Artmed, Porto Alegre, 2004.

SBC. SOCIEDADE BRASILEIRA DE CARDIOLOGIA; DHA. DEPARTAMENTO DE HIPERTENSÃO ARTERIAL. Tratamento da Hipertensão Arterial em Grupos Especiais, Negros e Miscigenados. 2002 Disponível em: <http://departamentos.cardiol.br/dha/consenso3//capitulo6 .asp.> Acesso em: 19.10.2015.

FREITAS, D.; RODRIGUES C. S.; YAGUI, C. M.; CARVALHO, R. S. T.; MARCHI, A. L. M. Fatores de risco para hipertensão arterial entre estudantes do ensino médio. 2012. Acta Paul. enferm., vol.25, n.3. 2012.

FONTANELLA, F. G.; GALATO, D.; REMOR, K. V. T. Perfil de automedicação em universitários dos cursos da área da saúde em uma instituição de ensino superior do sul do Brasil. Rev Bras Farm., Rio de Janeiro, v.94, n. 2, 2013.
GUPTA, S.; KAPOOR, S. Genetic and environmental influentces on blood pressure in na urban Indian population. Jornal BiosocSci. Cambridge, v. 45, n. 1, 2013.

GOULART, F. A. A. Doenças crônicas não transmissíveis: estratégias de controle e desafios e para os sistemas de saúde. Brasília: Ministério da Saúde, 2011. 92 p.

HDS. Hypertension in Diabetes Study. I. Prevalence of hypertension in newly presenting type 2 diabetic patients and the association with risk factors for cardiovascular and diabetic complications. J Hypertens, Estados Unidos, v. 11, n.6, Jun, 1993.

JACKSON, C.; HERBER-GAST, G.; BROWN, W. joint effects of physical activity and BMI on risk of hypertension in women: a longitudinal study. Journal of Obsity, New York, ID 271532, 2014

LIMA, V.; CAETANO, J. A.; SOARES, E.; SANTOS, Z. M. S. A. Fatores de risco associados a hipertensão arterial sistêmica em vitimas de acidente vascular cerebral. Rev Bras Promoç Saúde, Fortaleza, v. 19, n. 3, 2006.

LESSA I. Epidemiologia da insuficiência cardíaca e da hipertensão arterial sistêmica no Brasil. Rev Bras Hipertens, Salvador, v.8 p. 383-92, 2001.

LOPES, H. F. Genética e Hipertensão Arterial. Rev. Bras Hipertens, Rio de Janeiro, v. 21, n. 2, 2014.

LOPES,W. F. L.; COELHO, M. R. O. M.; OLIVEIRA, J.P.; ARAUJO, Y. M. O.; MELO, M. C. N.; TAPETY, F. I. A prática da automedicação entre estudantes de uma instituição de ensino superior de Teresina-PI. Rev. interdisciplinary. Teresina, v. 7, n. 1. 2014b.

MARTINS, M. C. C.; RICARTE, I. F.; ROCHA, C. H. L.; MAIA, R. B.; SILVA, V. B.; VERAS, A. B.; FILHO, M. D. S. Pressão Arterial, Excesso de Peso e Nível de Atividade Física em Estudantes de Universidade Pública. Rev .Arq Bras Cardiol. v. 95, n. 2, 2010.

MINAY, M .C. S.; GUALHANO, M. L. Vigilância de doenças crônicas e violências, o desafio contemporâneo

Rev. Ciênc. Saúde Coletiva, Rio de Janeiro v. 20, n.3, mar, 2015.

NETO, A. C. B.; ARAúJO, E. C.; SILVA, K. V. P.; PONTES, L. M. Prevalência de hipertensão e fatores associados em adolescentes escolares no sertão de Pernambuco. Rev. Adolesc Saude, Rio de Janeiro, v.7, n. 4, 2010 .

OLIVEIRA, J. R. W. M; GUSMÃO, J. L. Conhecimento sobre hipertensão arterial entre graduandos de enfermagem de uma universidade privada do município de Guarulhos-SP. Rev. Saúde, São Paulo, v.7, n 3/4, 2013. 
OMS. Organização Mundial Da Saúde. World health statistics 2011. World Health Organization. 2011. Acessado em: 15 de maio de 2016. Disponível em: < http://www.who.int/gho/publications/world_health_statisti cs/EN_WHS2011_Full.pdf?ua=1>

OMS. Organização Mundial Da Saúde. Doenças crônicas não transmissíveis causam 16 milhões de mortes prematuras todos os anos. 2014. Disponível em: $<$ http://www.paho.org/bra/index.php?option=com_content $\&$ view $=$ article $\&$ id=4766: doencas-cronicas-naotransmissiveis-causam-16-milhoes-de-mortes-prematurastodos-os-anos \&Itemid=839> Acesso em: 10 de maio de 2015 .

RADOVANOVIC, C. A. T.; BEVILAQUA, C. A.; AVELAR, F. S. R.; GIROTTO, P. C. M.; MARCON, S. $\mathrm{S}$. Prevalência de fatores de risco cardiovascular entre adolescentes universitários. Rev . Fiep Bulletin, Foz do Iguaçu, v. 82, 2012.

SANTOS, Z. M. S. A. Hipertensão Arterial - um problema de saúde pública. Rev. Bras. Promoção da Saúde, Fortaleza, v. 24, n. 4, Out/dez, 2011.

SILVA, L. S. F.; COSTA, A. M. D. D.; TERRA, F. D. S.; ZANETTI, H. H. V.; COSTA, R. D.; COSTA, M. D. Automedicação em acadêmicos de cursos de graduação da área da saúde de uma universidade privada do Sul do estado de Minas Gerais. Rev. Odontolclin cient. Camaragibe, v. 10, n. 1, 2011.

SILVA, E. S.S. B.; OLIVEIRA, S. F. S. B.; PIERIN, A. M.G. The control of hypertension in men and women: a analisy comparative. Rev Esc Enferm USP, São Paulo, v. 50, n.1, 2016.

SOAR, C.; SILVA, O. S.; LIRA, J. G. Consumo alimentar e atividade física de estudantes universitários da área de saúde. Rev. Univap, São José dos Campos, v. 18, n. 31, 2012.

SBC. Sociedade Brasileira de Cardiologia. In: Diretrizes Brasileiras de Hipertensão, 6. Arquivos Brasileiros de Cardiologia, São Paulo, v. 95, n. 1, s. 1. 2010.
SBC. Sociedade Brasileira de Cardiologia. Tratamento da Hipertensão Arterial em Grupos Especiais, Negros e Miscigenados, 2015. Disponível em: <http://departamentos.cardiol.br/dha/consenso3//capitulo6 .asp.> .Acessado em: 19 de outubro de 2015.

SBEM. Sociedade Brasileira de Endocrinologia e Metabologia. Dicas para prevenir Hipertensão, Disponível em: <http://www.endocrino.org.br/dicas-para-prevenirhipertensao/>. Acesso em: 19 de outubro de 2015.

SBH; SBC; SBN. Sociedade Brasileira de Hipertensão; Sociedade Brasileira de Cardiologia; Sociedade Brasileira de Nefrologia. In: Diretrizes Brasileiras de Hipertensão Arterial, 6. Rev. Bras. Hipertensão, v. 13, 2010.

SBH, Sociedade Brasileira de Hipertensão . Taxa de morte por hipertensão arterial cresceu $13,2 \%$ na última década. Acesso em: 18 de agosto de 2016. Disponível em: <http://www.sbh.org.br/geral/noticias.asp?id=486>.

UNITED NATIONS (UN). Resolutions adopted by the Genera 1 Assembly. 66/2. Political declaration of the High-Level Meeting of the General Assembly on the Prevention and Control of Non-Communicable Diseases, Washington: UN; 2011.

ZHANG, L.; QUI, L. Q.; LIU, A. P.; WANG, P. Y. Prevalence of risk factors for cardiovascular disease ant their ant their associations with diet and physical activity in Suburban Beijing. China. Journal of Epidemiology, Tokyo, v. 20, n. 3, 2010.

WESCHENFELDER, M., D.; GUE, M. J. Hipertensão arterial: principais fatores de risco modificáveis na estratégia. Enferm.glob. vol.11 n.26. 2012.

WHO. World Health Organization. Guideline: Sodium intake for adults and children, Geneva: WHO Library Cataloguing-in-Publication, 2012. Disponível em: < http://apps.who.int/iris/bitstream/10665/77985/1/9789241 504836_eng.pdf> Acessado em: 13 de agosto de 2016. 